\title{
Métrologie des systèmes optiques
}

\author{
P. Coussot
}

Matra, 17 rue Paul Dautier, F-78140 Velizy-Villacoublay, France

Le présent cours est la continuation logique de celui de Calcul Optique. Dans une première partie, à partir de la formule optique élaborée par ce dernier, il prend en compte, tout d'abord, l'établissement du bilan de tolérances, ce qui constituera le premier chapitre. Dans le second, il décrit les méthodes et les moyens de mesure à mettre en œuvre en cours de réalisation pour satisfaire ce bilan.

La seconde partie traite de la métrologie du système réalisé. Nous verrons donc les moyens d'évaluation de la qualité image d'un instrument d'optique, tels que la mesure de la distorsion et la FTM, ainsi que la mesure de certains paramètres particuliers comme la transmission et la lumière parasite.

\section{I.Metrologie du systeme en cours de fabrication}

\section{A) ETABLISSEMENT DES TOLERANCES}

\section{Tolérance sur la qualité de l'image}

Les paramètres qui peuvent dégrader la qualité du système optique sont classés en deux catćgorics : la première comporte les paramètres qui conservent le caractère de révolution du système optique, c'est-à-dire rayons de courbure, épaisseurs, intervalles et indices (en dehors de l'homogénéité). Ces paramètres sont potentiellement compensables les uns 
avec les autres. La seconde traite des paramètres qui ôtent au système ses propriétés de révolution. On y trouve les décentrements et inclinaisons de dioptres de lentilles simples ou de groupes collés. Les défauts d'irrégularité de surface entrent également dans cette catégorie. Ces paramètres ne sont pas compensables au sens strict du terme. Nous verrons cependant qu'il peut y avoir certaines compensations partielles, en décentrement par exemple.

Le cas particulier des surfaces asphériques, fort peu utilisées dans le domaine visible, mais souvent dans l'infra-rouge thermique 8-13 $\mu$, sera traité à titre indicatif dans un chapitre spécial, car le contrôle dépend étroitement du mode de fabrication et se fait la plupart du temps à ce niveau.

Tous ces paramètres, s'ils ne sont pas réalisés à leur valeur normale ou à la valeur zéro pour les excentrements, vont dégrader la qualité image théorique. Le but du calcul de tolérances est de déterminer la variation possible sur chacun des paramètres, de manière à ce que l'image finale ne subisse pas une dégradation supérieure à une valeur prévue. Le concepteur et calculateur du système optique est responsable de la qualité image du système réalisé. Le calcul du bilan de tolérances est donc une part importante de son travail et, à ce titre, a déjà été abordé dans le cours précédent. Nous compléterons ici par l'étude d'un logiciel TOLERANCE de CODE V, qui permet d'obtenir aisément ce bilan, et qui fournit en outre l'évaluation des performances finales du système.

Il est important de noter que ce calcul des tolérances, nécessaire à la fin de l'étude optique, peut également être un support de celle-ci. En effet, la détermination de la sensibilité des différents paramètres peut orienter le choix de la conception de la formule optique et, en particulier, celui du nombre et de la position des lentilles. Il peut être plus économique de réaliser un objectif de six lentilles centrées à $10 \mu \mathrm{m}$ qu'un objectif de quatre lentilles centrées à 1 ou $2 \mu \mathrm{m}$.

Avant d'entreprendre ce bilan, on peut éliminer des paramètres potentiels ceux qui sont compensables plus ou moins facilement. Par exemple, il est très courant de refaire un calcul dit $\mathrm{d}^{\prime \prime}$ adaptation aux fontes", avec les valeurs d'indices fournies par le verrier. Le paramètre indice peut être ainsi considéré à la tolérance zéro. De même, en cours de réalisation, on peut mesurer les épaisseurs des lentilles effectivement réalisées, et on compense par quelques intervalles d'air. Ceci permet d'exclure les épaisseurs du bilan de tolérances —ou, tout au moins, de les 


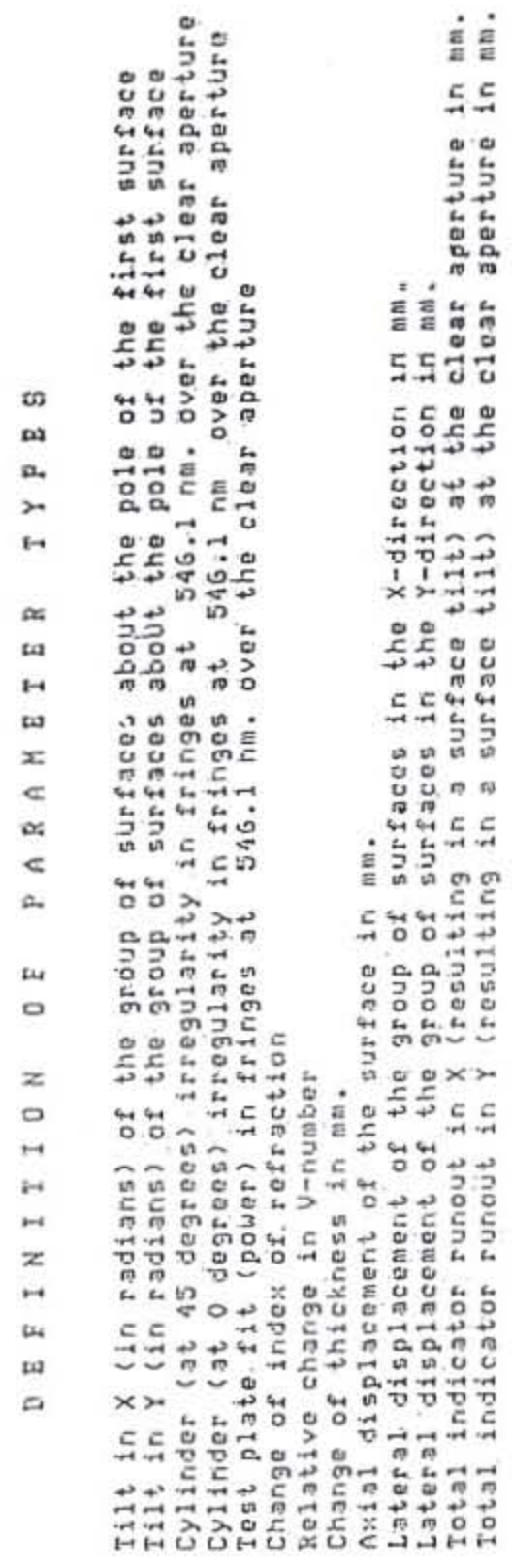

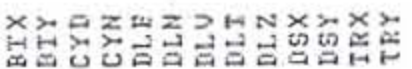


limiter à des valeurs que l'on obtient couramment en usinage- et de faciliter ainsi la fabrication des lentilles.

Cette sélection des paramètres étant faite, on choisit un critère d'évaluation : CODE $\mathrm{V}$ donne le choix entre une FTM à une fréquence donnée, ou un écart moyen à la surface d'onde "RMS" (Root Mean Square $\left.=1 / \mathrm{n} \sqrt{ }\left(\sum \mathrm{x}_{\mathrm{i}}{ }^{2}\right)\right)$. Le choix est fait en fonction des spécifications de la fourniture, et le critère de FTM est choisi dans huit cas sur dix.. L'expérience montre que celui-ci est souvent plus sévère ; en effet, il tient mieux compte de la structure de l'image $(\lambda / 5$ d'aberration sphérique dégrade moins la FTM que $\lambda / 5$ de coma). Dans la plupart des cas, on évalue la dégradation dans le meilleur plan image, c'est-à-dire avec refocalisation. Il est évident que, si l'on veut conserver la qualité dans un plan image fixe, les tolérances seront beaucoup plus sévères.

Compte tenu de ces considérations, on établit par CODE $\mathrm{V}$ un tableau donnant l'influence sur la FTM, à la fréquence choisie, des différents paramètres restant susceptibles de varier. Ce tableau est fait pour deux ou trois valeurs de champ, l'axe et le plus grand champ étant souvent suffisants. Pour ce dernier, on peut se contenter de la dégradation tangentielle qui est, dans la plupart des cas, la plus importante. A titre d'exemple, on donne ci-après plusieurs tableaux : les deux premiers (tableaux I et II) présentent l'influence sur la FTM d'une variation de divers paramètres: rayons de courbure (exprimés en franges), décentrements (DSX ou DSY), et inclinaisons (BTX et BTY). Les deux suivants (tableaux III et IV) montrent une formule optique avec ses paramètres, ainsi que leurs tolérances géométriques et de décentrement. A la suite de ces tableaux sont donnés les probabilités de dégradation de la FTM. Le programme détermine ces chiffres par un calcul de probabilités basé sur la méthode dite de "Monte Carlo".

Il est souvent intéressant de classer ces tolérances par ordre d'importance sur la dégradation de FTM. Le programme fait automatiquement ce travail, et on obtient le tableau V. Compte tenu des possibilités de réalisation, on peut aussi agir sur chaque paramètre en lui donnant sa tolérance réelle, et en tirer immédiatement les conséquences probables sur la FTM. Par exemple, nous avons trouvé dans l'exemple précédent que les rayons de courbure avaient une tolérance de trois franges. Or les méthodes de fabrication permettent d'avoir pratiquement une frange. Nous pouvons donc remplacer, dans le tableau $\mathrm{V}$, la tolérance 


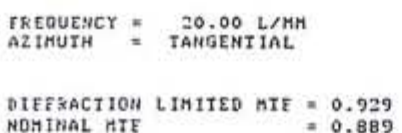

Tableaux I et II $c=0.889240$

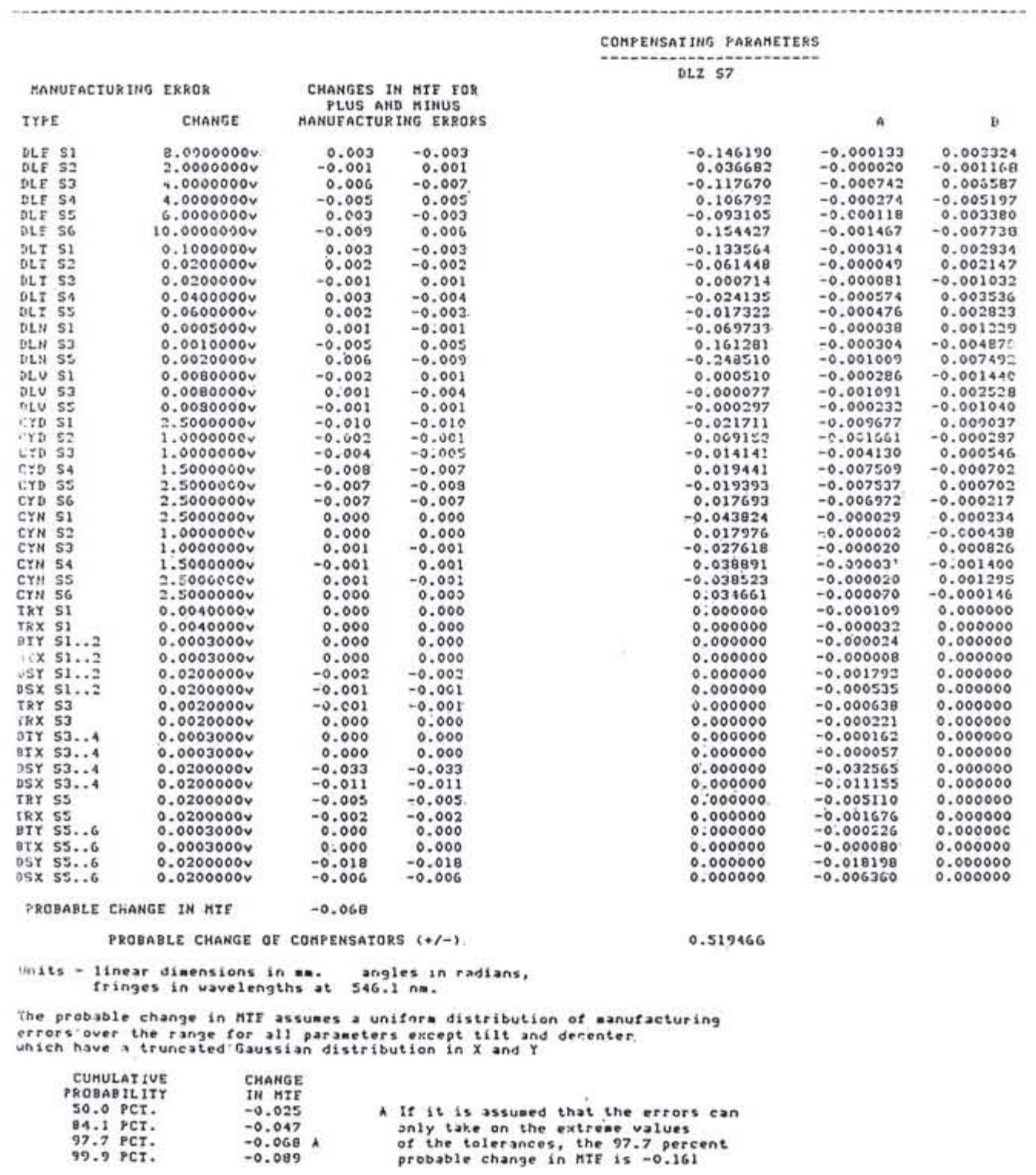


Tableaux III et IV

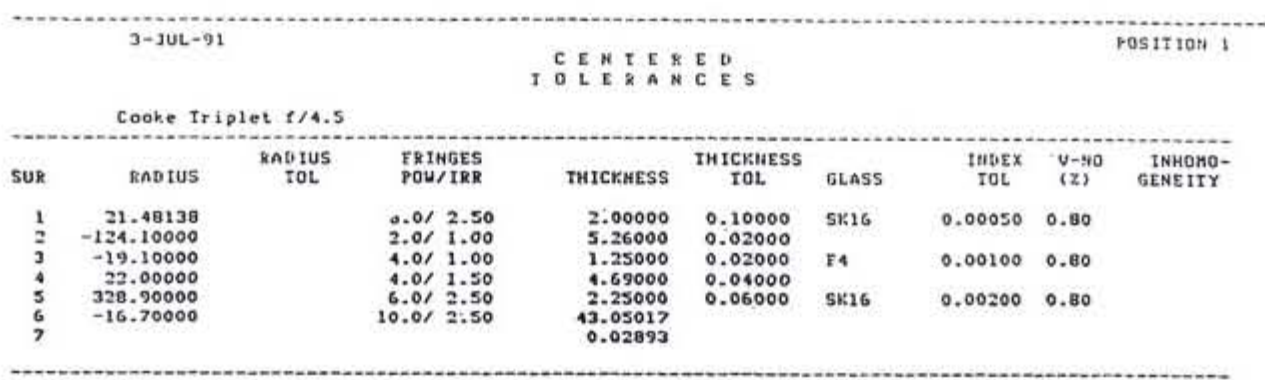

Radius, radius tolerance, thiekness and thickness tolerance are given in $\mathrm{ma}$.

Eringes of power and irregularity are at $596.1 \mathrm{na}$. over the clear aperture

trregularity is defined as fringes of cylinider power in test plate fit

\begin{tabular}{|c|c|c|c|c|c|c|c|c|}
\hline \multirow[b]{2}{*}{$\begin{array}{l}\text { TLEMENT } \\
\text { NO. }\end{array}$} & \multicolumn{8}{|c|}{ 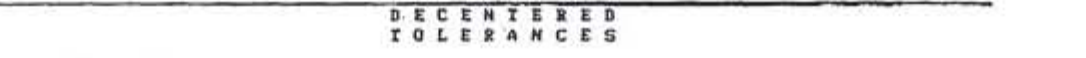 } \\
\hline & $\begin{array}{l}\text { ERONT } \\
\text { RADIUS }\end{array}$ & $\begin{array}{l}\text { BACK } \\
\text { RADIUS }\end{array}$ & $\underset{\text { IIR }}{\text { ELE }}$ & $\begin{array}{l}\text { IT UEDGE } \\
\text { ARC NIN }\end{array}$ & $\begin{array}{l}\text { ELE } \\
\text { IIR }\end{array}$ & $\begin{array}{l}\mathrm{TILT} \\
\mathrm{ARC} \mathrm{HIN}\end{array}$ & $\underset{T I R}{E L}$ & DEC/ROLLC(R) \\
\hline $\begin{array}{l}1 \\
3 \\
3\end{array}$ & $\begin{array}{r}21.48138 \\
-19.10000 \\
328.90000\end{array}$ & $\begin{array}{r}-124.10000 \\
22.00000 \\
-16.70000\end{array}$ & $\begin{array}{l}0.0010 \\
0.0020 \\
0.0200\end{array}$ & $\begin{array}{l}1.0 \\
0.8 \\
5.3\end{array}$ & $\begin{array}{l}0.0041 \\
0.0027 \\
0.0039\end{array}$ & $\begin{array}{l}1.0 \\
1.0 \\
1.0\end{array}$ & $\begin{array}{l}0.0152 \\
0.0179 \\
0.0169\end{array}$ & $\begin{array}{l}0.0200 \\
0.0200 \\
0.0200\end{array}$ \\
\hline
\end{tabular}

Radii are given in units of $m$.

For vedge and tilt, TIR is a-single indicator masureant taken at the saaller

the induced wedge and is the maximus ditrerence in readings between two

indicators, one for each surface, with both surfaces masured at their respective clear apertures. oria is aeasured in a..

Decenter or roll is measured perpendicular to the optical axis, in ma-

\begin{tabular}{|c|c|c|c|c|c|c|}
\hline $\begin{array}{l}\text { RELATIVE } \\
\text { EIELD }\end{array}$ & $\begin{array}{l}\text { EREO } \\
\mathrm{L} / \mathrm{MH}\end{array}$ & $\underset{\text { DEG }}{\text { AZIM }}$ & WEIGHT & DESIGM & $\begin{array}{l}\text { DESIGN } \\
+ \text { TOL }\end{array}$ & $\begin{array}{l}\text { COMPENSATOR RANGE }(+/-) N^{*} \\
\text { DLZ, S7 }\end{array}$ \\
\hline $\begin{array}{ll}0.00, & 0.00 \\
0.00 ; & 0.69 \\
0.00, & 1.00\end{array}$ & $\begin{array}{l}20.00 \\
20.00 \\
20.00\end{array}$ & $\begin{array}{l}\text { TAN } \\
\text { TAM } \\
\text { TAN }\end{array}$ & $\begin{array}{l}1.00 \\
1.00 \\
1.00\end{array}$ & $\begin{array}{l}0.889 \\
0.426 \\
0.277\end{array}$ & $\begin{array}{l}0.821 \\
0.369 \\
0.234\end{array}$ & $\begin{array}{l}0.519466 \\
0.519466 \\
0.519466\end{array}$ \\
\hline
\end{tabular}

t The change in hie is a mean blus 2 51gaa-value (97.7. percent)

and assumes a unifora.distribution of manuracturing errors over the range Gaussian distribution in $x$ and $y$

The compensator range is a wean plus 2 . S1gma value.

Anguiar compensators are in radians. 


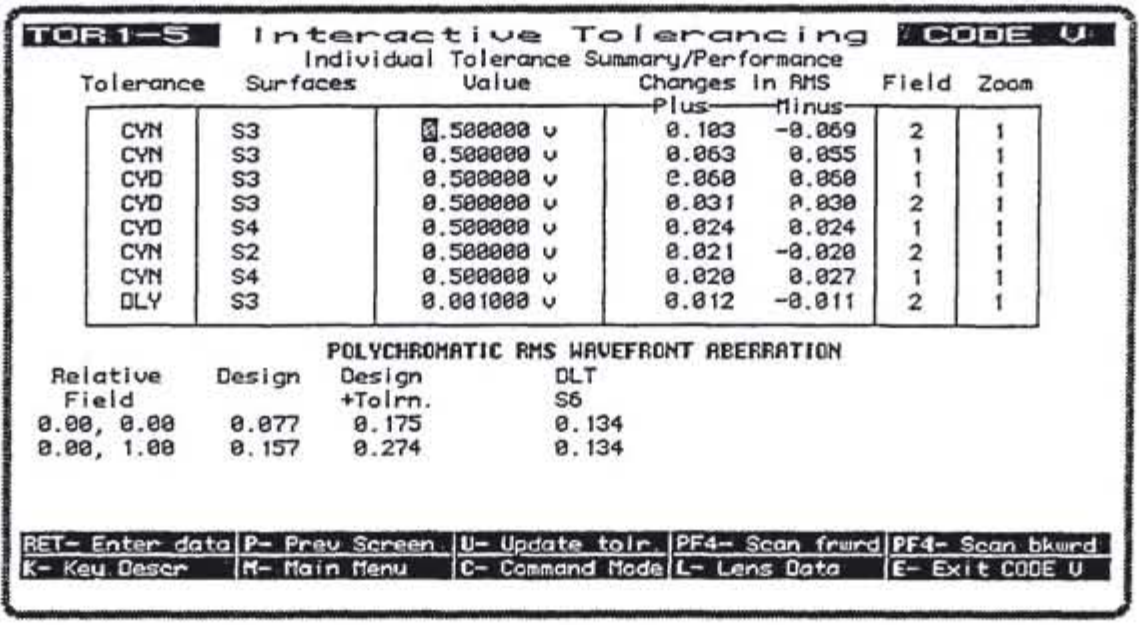

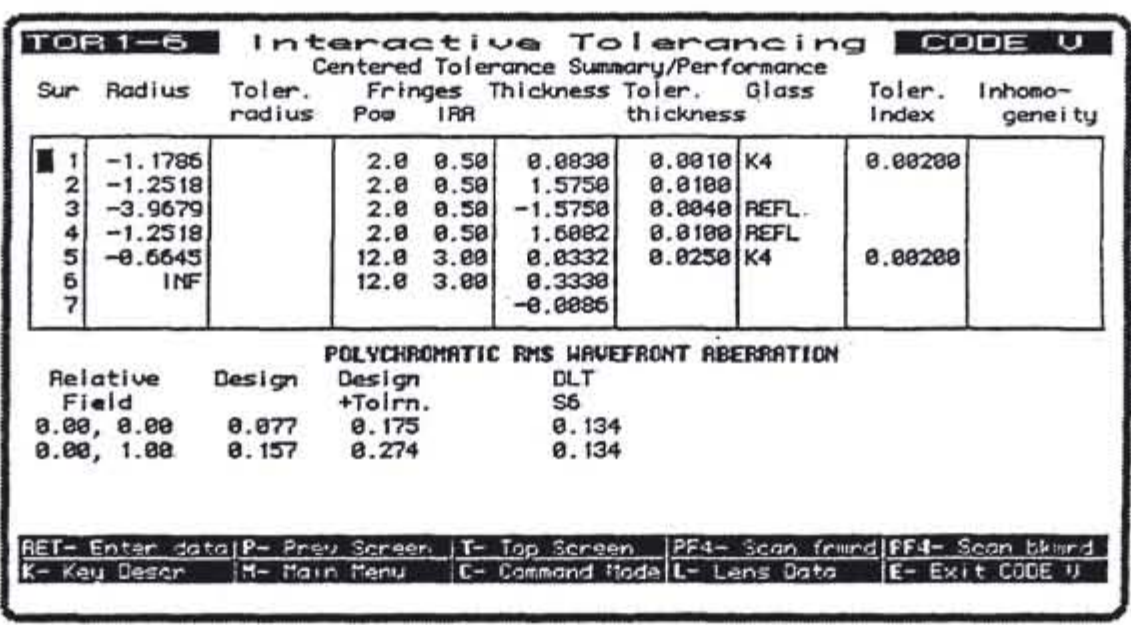


de trois franges par celle d'une frange sur chaque rayon, et nous aurons immédiatement l'évaluation du gain sur la FTM.

\section{Tolérances sur la distorsion}

La distorsion est une aberration de grandissement et, de ce fait, indépendante de la qualité image. Les tolérances calculées précédemment n'ont donc aucune signification pour elle. Il faut faire un calcul particulier pour obtenir l'influence sur la distorsion de chacun des paramètres. Ce travail n'est fait que pour les systèmes où la distorsion est fort importante, c'est-à-dire les grands angulaires, ou ceux où il est nécessaire d'avoir une carte de distorsion extrêmement précise, tels que les objectifs de microlithographie, où la grandeur de l'image doit être connue à quelques centièmes de microns près. Le tableau VI obtenu donne l'influence de chaque paramètre sur la distorsion, ainsi que la probabilité de dégradation de cette dernière en fonction de tous les paramètres. Ces deux tableaux (V et VI) indépendants doivent être associés pour créer un tableau VII, donnant les tolérances permettant de satisfaire à la fois les conditions de qualité image et de distorsion.

Nous sommes amenés maintenant à décrire les divers moyens qui sont actuellement utilisés pour fabriquer et monter les éléments du système optique en respectant les tolérances. En parallèle, nous aborderons les moyens de contrôle, c'est-à-dire la métrologie du système optique en cours de réalisation. Nous allons suivre chronologiquement toutes les phases d'une fabrication, en commençant par l'approvisionnement des matériaux, puis le polissage des verres, le débordage et enfin le montage. 
Tableau VI

Output: Distortion Table (not all tolerances shown)

CODE $V$

Position 1

DISTORTION SENSITIVITY

Triplet U,s, patent 2,615,157

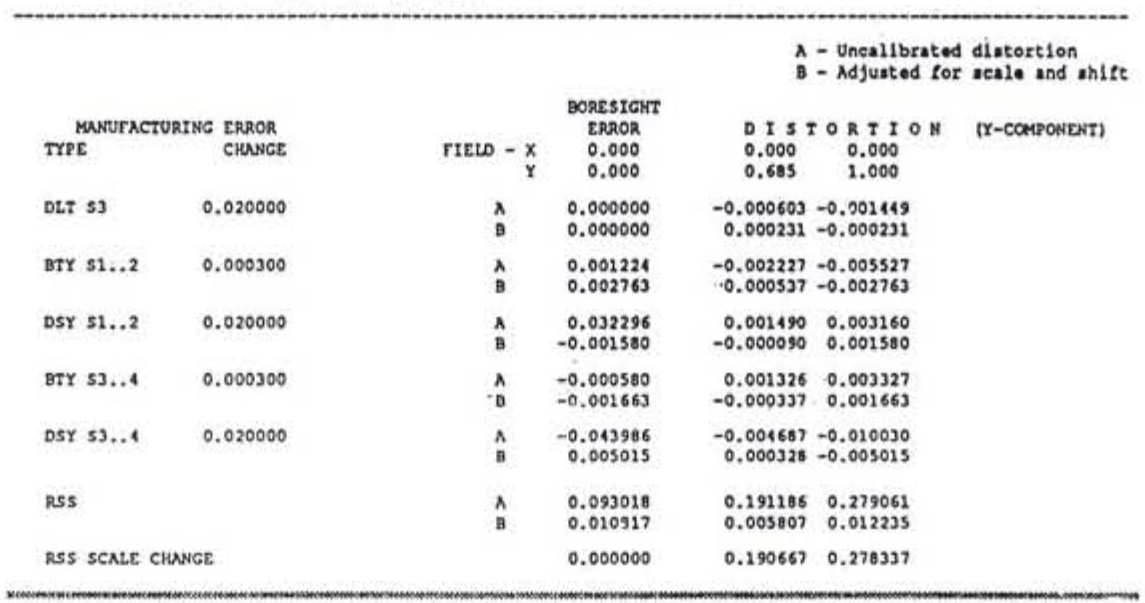




\section{B) APPROVISIONNEMENT ET CONTROLE DES MATERIAUX OPTIOUES}

L'approvisionnement des matériaux optiques est déterminé par l'ingénieur responsable du calcul optique. Leur nature dépend de la bande spectrale utilisée. Pour le visible et le proche infra-rouge 400 à $2000 \mathrm{~nm}$, nous employons les verres optiques fournis par les grands verriers mondiaux SCHOTT, CORNING, OHARA et HOYA. Chacun a un catalogue dans lequel les ingénieurs calculateurs peuvent consulter toutes les caractéristiques de chaque verre, c'est-à-dire la valeur des indices pour les longueurs d'onde correspondant à des raies spectrales fixes, ainsi que les moyens d'interpolation pour calculer l'indice à chaque longueur d'onde. Notons que ces données sont entrées dans la plupart des programmes modernes de calcul. Lors de la commande des verres, l'ingénieur précisera la qualité d'homogénéité dont il a besoin; les qualités vont de standard $(\mathrm{dn}=2.10-5)$ à $\mathrm{H} 4\left(\mathrm{dn}=2 \cdot 10^{-5}\right)$. Il peut également préciser la biréfringence acceptable, ainsi que la qualité en tant que bulles et stries. Un dernier paramètre, qui est demandé de plus en plus fréquemment, est le coefficient de variation thermique de l'indice, $B=d n / d t$. En effet, on spécifie une plage de température de fonctionnement assez large qui nécessite de choisir des verres spécifiques, dits athermaux. En première approximation, à partir de la relation :

$$
1 / F=(n-1) / R
$$

pour une lentille simple plan convexe, on doit avoir :

$$
\mathrm{dF} / \mathrm{F}=\mathrm{dn} /(\mathrm{n}-1)-\mathrm{dR} / \mathrm{R}
$$

ce qui nous donne, si l'on veut $\mathrm{dF}=0$ pour une variation $\mathrm{dt}$ de température :

$$
B /(n-1)=\alpha
$$

$\alpha$ étant le coefficient de dilatation du verre.

L'examen des catalogues des verriers montre qu'il existe un certain nombre de verres qui satisfont approximativement cette relation et qui peuvent être considérés intrinsèquement comme athermaux. Pour d'autres verres ayant des $\mathrm{dn} / \mathrm{dt}$ négatifs, les deux termes s'ajoutent dans la relation précédente et la sensibilité thermique de ces matériaux est grande. C'est le cas de la plupart des verres favorables à la correction de l'apochromatisme. Si le fonctionnement de tels objectifs doit se faire sur 
une grande plage de température, il sera nécessaire de prévoir un dispositif de refocalisation actif ou passif.

Le coefficient $B$ des verres optiques varie de $-10.10-6$ à $+12.10-6$, ce qui n'est pas très important en regard des matériaux infra-rouge, où le germanium a un dn/dt de $400.10^{-6}$, le silicium de $160.10^{-6}$ et le $\mathrm{ZnS}$ de 43.10-6. Ces chiffres font que de tels systèmes ne peuvent être utilisés qu'en température stabilisée, ce qui nécessite des dispositifs complexes de climatisation avec contrôle de la température. A titre d'exemple, une lentille en germanium de focale $100 \mathrm{~nm}$ a une variation de 12 microns par degré. La recherche de systèmes infra-rouge athermaux passifs est d'une grande actualité. Elle est souvent liée à l'utilisation de nouveaux matériaux IR tel que les AMTIR ou les calchogénures.

\section{C) USINAGE ET CONTROIE DES SURFACE OPTIOUES SPHERIOUES}

Les matériaux optiques sont livrés sous formes de blocs, de barres ou de disques pré-formés. Selon le mode de livraison, la première opération de l'usinage, l'ébauchage, est plus ou moins longue. Celui-ci, en effet, consiste en opérations de sciage, découpage et usinage avec un émeri grossier, opérations destinés à mettre en forme approchée (en épaisseur et en rayon de courbure) les éléments optiques.

La seconde opération est le polissage. Le fabricant dispose pour les rayons de courbure à réaliser d'outils sphériques concaves ou convexes, généralement en laiton, dont le rayon de courbure est égal à celui à obtenir. Il dispose en outre d'une paire de calibres convexe et concave à la valeur exacte mesurée à l'interféromètre avec une grande précision. L'art du polisseur consiste, en usant la surface avec des émeris de plus en plus fins, sur l'outil tournant, à obtenir une surface sphérique polie régulière ayant le rayon de courbure souhaité. Cette opération peut se faire sur un "tour à pédales", le polisseur assurant alors lui-même la rotation de l'outil avec ses pieds, tandis qu'il tient dans sa main l'élément 
à polir. Mais elle est maintenant réalisée sur des machines automatiques, où l'homme n'intervient que dans les réglages préliminaires et dans les contrôles en cours de fabrication.

Ceux-ci se font traditionnellement par la méthode du "calibrage"; le polisseur pose son calibre sur l'élément optique très soigneusement nettoyé au préalable, et fait apparaître des franges de coin d'air entre les deux surfaces. Selon ces franges, il réglera sa machine pour obtenir une surface plus convexe ou plus concave. Lorsque les rayons sont identiques, il obtient la "teinte plate". Naturellement, pour des raisons économiques évidentes, on cherchera toujours à polir plusieurs lentilles à la fois. Pour cela, les lentilles sont fixées sur un bloc par de la poix, et on peut ainsi polir la surface de plusieurs dizaines d'éléments en même temps. Il existe également, depuis quelques années, des techniques de polissage rapide basées sur un principe différent et permettant de réduire considérablement les temps de polissage. Ceux-ci dépendent essentiellement de deux paramètres : la précision recherchée sur la surface et le diamètre de celle-ci. Ces temps, que nous donnons à titre d'exemple, sont difficilement comparables, car ils peuvent être dans un rapport de mille (entre une lentille de $\emptyset 40$ à $\lambda / 2$ en polissage rapide et une lentille de $\emptyset 350$ faite individuellement à $\lambda / 10$ ) :

Dans les cas de fabrications de séries, ce contrôle aux calibres est suffisant, mais pour les prototypes très performants, il faut procéder à un contrôle de la surface et à la mesure du rayon à l'interféromètre.

Les tests par interférométrie optique sont utilisés depuis longtemps en métrologie optique. Un interféromètre optique est un instrument de mesure qui utilise le phénomène d'interférences, lié à la différence de marche entre une onde de référence et une onde de mesure. Ces ondes, en se recombinant, forment des franges d'interférences. Les propriétés géométriques de celles-ci sont fonctions des différences de chemin optique entre les deux ondes (cf schéma ci-après). Ce procédé connu depuis très longtemps dans son principe souffrait d'un handicap important dès que la 

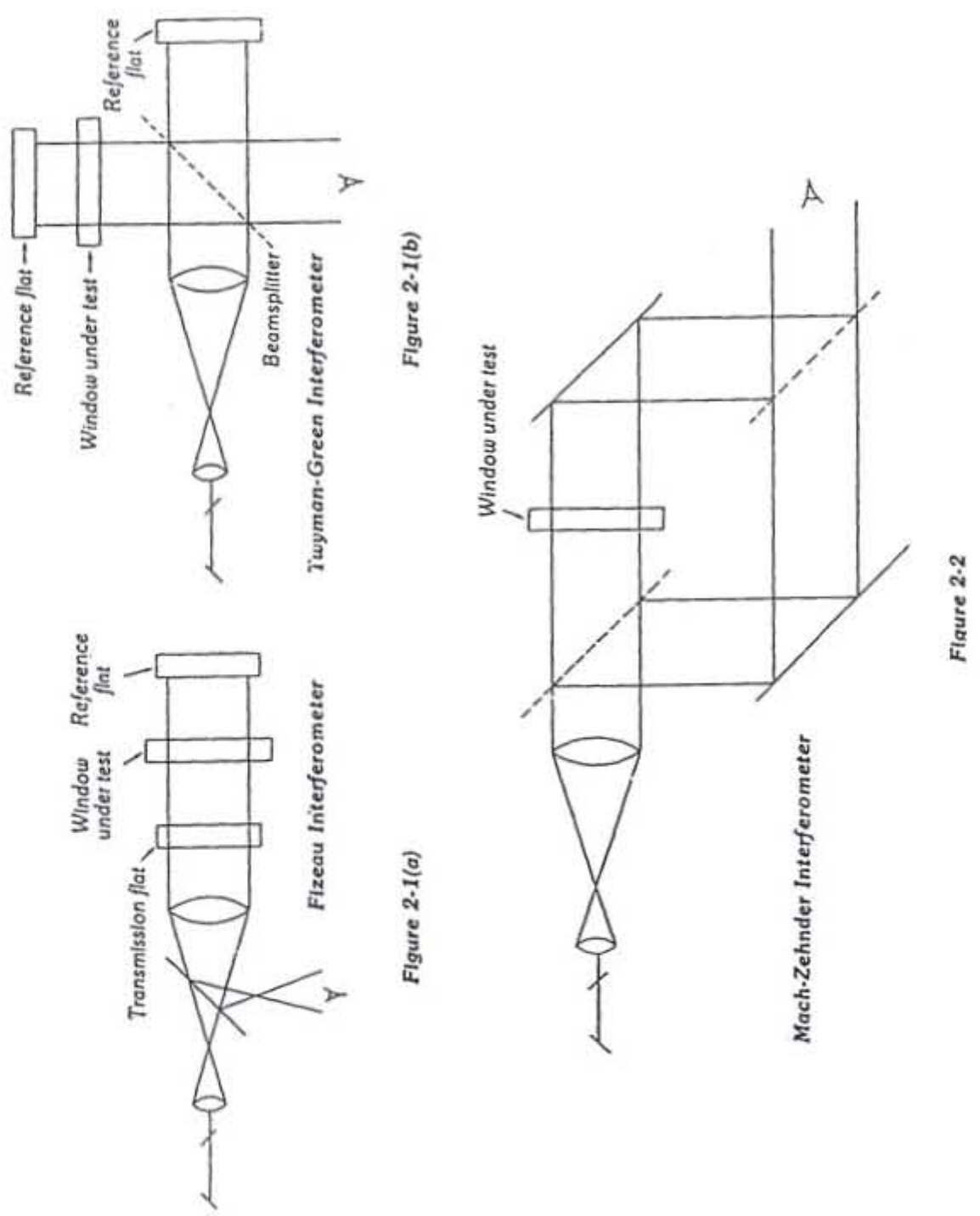
différence de marche était grande, du fait du manque de cohérence des sources utilisées. L'arrivée du laser, source monochromatique et cohérente par excellence, a donné un nouvel intérêt à l'utilisation des interféromètres. Ceux-ci sont maintenant des outils courants, et chaque fabricant d'optique a donc à sa disposition un ou plusieurs instruments de mesure de ce type. Ces interféromètres peuvent être utilisés aussi bien en cours de réalisation, pour établir la qualité de surface des éléments pris individuellement, qu'au stade final, pour déterminer un écart à la surface d'onde du système complet représenté par un interférogramme. On peut même, en introduisant ce dernier dans le programme de tolérances, refaire une "optimisation" du système sur les paramètres qui restent disponibles, tels que les intervalles d'air, les centrages, ou les inclinaisons par exemple.

Nous décrirons en annexe 2, de manière plus détaillée, l'interféromètre ZYGO, qui est en usage dans toute l'industrie optique.

\section{D) TRAITEMENT DE SURFACE}

Avant de voir la méthode de mesure des épaisseurs, nous allons poursuivre le cycle de fabrication des éléments optiques. Ceux-ci sont maintenant polis, et ont des surfaces réalisées dans les tolérances. Les deux opérations suivantes sont le débordage optique et le traitement de surface. Elles peuvent se faire dans un ordre quelconque. Le débordage optique étant cependant très variable en précision selon les méthodes de montage utilisées, nous l'aborderons en même temps que le débordage mécanique et le montage.

Le traitement de surface des matériaux optiques a pour but de minimiser le plus possible la réflexion sur chaque surface air-verre, qui a deux conséquences importantes: la perte en transmission, donc en éclairement de l'image, et la présence de lumière parasite. Rappelons que le coefficient de réflexion s'exprime en incidence normale par la relation : 


$$
\mathrm{R}=(\mathrm{n}-1) 2 /(\mathrm{n}+1)^{2}
$$

Cette formule donne les $4 \%$ bien connus pour l'indice 1,5 , mais elle donne également $8,16 \%$ pour l'indice 1,8 et $36 \%$ pour le germanium d'indice 4. Le traitement untiréfléchissant est donc une nécessité, et il n'existe pratiquement plus d'instruments non traités. L'objet de ce cours n'est pas d'entrer dans les détails des traitements multicouches très performants qui sont couramment utilisés par les spécialistes. On peut se souvenir de $\sqrt{n}$ comme indice du matériau de la simple couche d'épaisseur $\lambda / 2$ à évaporer sur un indice $\mathrm{n}$ pour obtenir une réflexion égale à zéro pour $\lambda$. Ces traitements se font sous vide, généralement à hautes températures, par évaporation ou par canon à électrons. Dans le cas où les précisions de surfaces demandées sont très performantes, l'opération de traitement peut détériorer légèrement la qualité obtenue avant traitement. Ce phénomène est particulièrement sensible pour les diamètres importants. Dans ce cas, il est souhaitable de refaire un mesure à l'interféromètre pour vérifier la qualité de surface après traitement.

La mesure des épaisseurs peut se faire à ce stade. Une méthode simple consiste à utiliser un pied à coulisses qui nous donnera une précision de 1/100 de $\mathrm{mm}$. Si cela est insuffisant, nous pouvons utiliser un procédé plus élaboré, basé sur l'emploi de comparateurs, qui nous permettra d'atteindre des précisions de l'ordre du micron. Certains fabricants ont réalisé pour leur propre compte une machine automatique qui fournit un résultat quasi-instantané et d'une très grande précision.

\section{E) DEBORDAGE ET CENTRAGE DES ELEMENTS}

\section{OPTIOUES}

Les lentilles étant usinées, traitées et souvent pré-débordées, nous allons aborder la phase très importante de l'association des éléments optiques avec la mécanique de manière à réaliser un ensemble ou un sous-ensemble complet. Cette opération fondamentale a beaucoup évolué depuis une cinquantaine d'années, car elle a suivi les progrès 
considérables qui ont été faits dans le domaine du "calcul optique ", avec l'arrivée des ordinateurs et des logiciels correspondants.

Nous allons examiner les diverses méthodes, de la plus simple à la plus complexe, et nous donnerons à chaque fois les tolérances que nous pouvons espérer atteindre.

La méthode la plus simple et la plus ancienne est l' "empilage”. Les lentilles sont débordées optiquement de manière à ce que l'axe optique soit confondu avec l'axe mécanique (matérialisé par le diamètre extérieur des lentilles). La vérification était faite traditionnellement avec un filament de lampe, dont on observait l'image sur une face de la lentille; la deuxième face de cette dernière étant placée sur un cylindre fixé à l'axe d'un tour en rotation, l'image de retour décrit un cercle lorsque les deux axes ne sont pas confondus. On rectifiait le débordage directement sur le tour, de façon à ce que cette image de retour soit fixe.

Les lentilles ainsi débordées sont ensuite placées dans une monture, des entretoises intercalées entre chaque lentille permettant de respecter les intervalles entre elles. La première lentille s'appuie sur la base de la monture, et une bague fileté à l'arrière fixe l'ensemble. Dans ce type de montage, compte tenu des défauts résiduels de débordage et du jeu nécessaire au montage, on ne peut pas espérer des centrages meilleurs que $0,03 \mathrm{~mm}$, et proches en moyenne de $0,05 \mathrm{~mm}$.

Le sertissage, pratiquement inutilisé aujourd'hui, était une variante de cette méthode, donnant à peu près la même précision. Chaque lentille était placée contre la face d'appui d'un barillet de métal, l'épaisseur suffisamment mince de ce barillet permettant de rabattre une languette de métal sur toute la circonférence de la lentille, qui se trouvait ainsi enserrée dans son barillet. Le sertisseur, qui faisait cette opération sur un tour, centrait "à la lumière ", en confondant au mieux l'axe mécanique du barillet et l'axe optique de la lentille. Cette opération était particulièrement délicate, seuls quelques spécialistes parvenaient à obtenir des résultats remarquables. De plus, l'inconvénient de cette méthode était qu'avec le temps, le sertissage se desserrait légèrement et que, les lentilles ayant la possibilité de bouger dans leur monture, l'objectif se déréglait et pouvait même se casser dans des cas exceptionnels.

Nous allons maintenant étudier les procédés modernes de débordage-centrage :

a) entre cloches 
La lentille à déborder est placée entre deux cylindres, sur un tour en rotation. Lorsque les surfaces sphériques sont centrées sur les cylindres, on déborde le diamètre extérieur de la lentille, et on a ainsi l'axe des dioptres confondu avec l'axe mécanique. La précision du procédé dépend de la courbure du rayon.

b) centrage laser

La lentille est placée sur un cylindre en rotation, et est fixée sur ce cylindre par aspiration. Un faisceau laser coaxial au tour traverse la lentille, et son impact sur un détecteur est visionné sur un moniteur TV. Lorsque les axes des deux dioptres ne sont pas confondus, le faisceau laser est dévié et décrit un cercle. En faisant glisser la lentille sur le cylindre, on réduit le cercle à un point, et on déborde par une meule diamant le diamètre de la lentille. Ce procédé est beaucoup plus précis que le précédent.

c) mécanique avec comparateur

Un cylindre est monté sur un tour. On vérifie à l'aide d'un comparateur que le plan de référence du cylindre est parfaitement perpendiculaire à l'axe optique. Si ce n'est pas le cas, une passe d'usinage de finition assure ce réglage. Le cylindre n'est plus démonté, la lentille est collée à chaud en appui sur le cylindre, donc le centre de ce dioptre passe par l'axe de ce cylindre. On place un comparateur sur la deuxième face qui peut, lorsque la lentille toume, enregistrer des battements de quelques dixièmes de microns. Pour centrer le deuxième dioptre, on fait glisser (après réchauffage de la colle) le premier dioptre sur le cylindre pour réduire à zéro le battement du comparateur. Une fois centré, on déborde avec la meule diamantée par passes successives. Cette méthode permet des précisions de centrage au micron.

Toutes ces méthodes décrites pour une lentille nue peuvent s'adapter à des lentilles collées dans un barillet. On procède alors au débordage du barillet par un outil de tour. 
204 P. Coussot

$-a$.

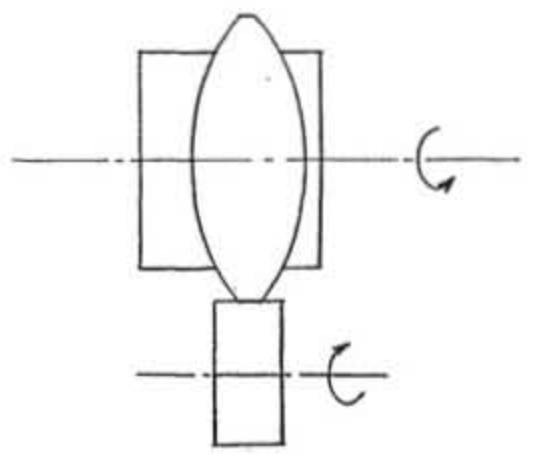

$-b-$

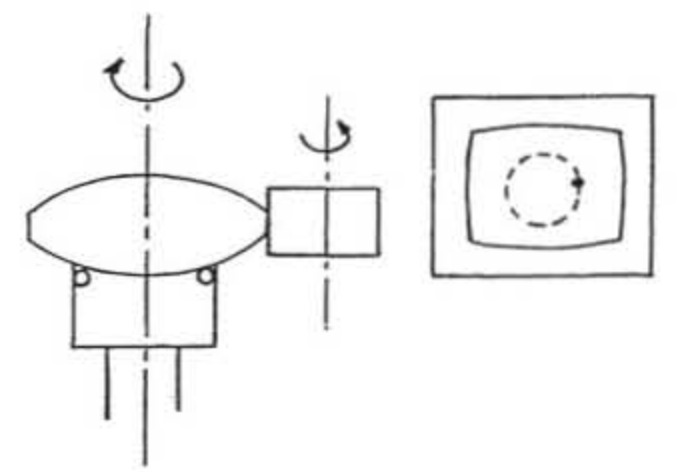

$-c$ -

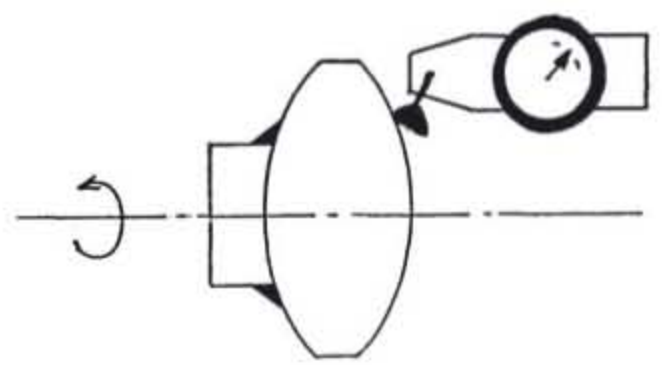




\section{Metrologie du systeme realise}

\section{A) EVALUATION DE LA OUALTTE DE L'IMAGE}

Nous allons maintenant aborder le contrôle final de la qualité de l'objectif ou de linstrument d'optique, dans sa forme définitive. La méthode de base était l'observation de l'image d'un point lumineux placé au foyer d'un collimateur, ceci permettant de voir les aberrations résiduelles sous leur forme théorique : cercle d'aberration sphérique, aigrette de coma, focales d'astigmatisme sagittale et tangentielle. Cette méthode est encore utilisée aujourd'hui pour mesurer le chromatisme longitudinal ou pour évaluer qualitativement un système optique.

Après cette opération, qui ne permet pas de "chiffrer" la qualité de l'instrument, on passait à la mesure du pouvoir séparateur. Des mires de Foucault à différentes fréquences étaient placées au foyer d'un collimateur, et on observait visuellement ou photographiquement sur l'image quelle était la fréquence la plus élevée pour laquelle on "séparait" les traits blancs des traits noirs. Cette méthode permettait de donner un chiffre à l'objectif ( 50 traits par $\mathrm{mm}$, par exemple), mais elle présentait certains inconvénients, dont le principal était l'interprétation de la "séparation", souvent différente chez le client et le fournisseur de l'objectif.

L'arrivée de la FTM et des instruments de mesure de celle-ci ont permis de donner des chiffres indiscutables quantifiant la qualité d'un instrument, puisque, pour chaque fréquence, on a la valeur du contraste de l'image, et l'on peut comparer la courbe obtenue pratiquement à celle déterminée théoriquement par le calcul.

Nous allons examiner plus en détail les méthodes de mesure de FTM et les appareils correspondants. Il faut cependant remarquer que cette évaluation par la FTM, qui est universellement adoptée pour les instruments usuels ou à film photographique, a subi une légère défaveur depuis l'arrivée des détecteurs et des "plans focaux". Dans ce cas, le 
critère de qualité est le pourcentage d'énergie qui entre dans un diamètre donné, en tenant compte à la fois des aberrations et de la diffraction.

\section{Définitions normalisées}

\section{1) PSF, LSE, E,SF}

\subsection{1-PSF}

La fonction d'étalement d'un point (PSF, de l'anglais Point Spread Function) est une distribution proportionnelle à la distribution $\mathrm{D}(\mathrm{x}, \mathrm{y})$ d'éclairement dans l'image d'un objet ponctuel, définie de telle sorte que son intégrale sur toute la surface réceptrice soit égale à l'unité :

$$
\operatorname{PSF}(x, y)=\frac{D(x, y)}{\iint_{\infty} D(x, y) d x \cdot d y}
$$

La PSF a donc la dimension de l'inverse du carré d'une variable d'espace.

\subsection{2- LSF}

La fonction d'étalement d'une ligne (LSF, de l'anglais Line Spread Function) représente la répartition du flux dans l'image d'une ligne droite. C'est une distribution que par définition on relie à la PSF :

$$
\operatorname{LSF}(x)=\int_{-\infty}^{+\infty} \operatorname{PSF}(x, y) d y
$$

La LSF a donc la dimension de l'inverse d'une variable d'espace.

\subsection{3- ESF}

La fonction d'étalement d'un bord de plage (ESF, de l'anglais Edge Spread Function) représente la répartition du flux dans l'image d'un objet constitué de deux plages se limitant mutuellement le long d'une droite, dont l'une a une luminance nulle. C'est une distribution que, par définition, on relie à l'ESF de la manière suivante :

$$
\operatorname{ESF}(x)=\int_{-\infty}^{x} L S F(t) d t
$$

Cette distribution est donc sans dimension. 


\section{2) FTO, FTM}

1.2.1. FTO (Fonction de Transfert optique)

Par définition, et même si la tache image d'un point dépend de la position du point objet, on définit la fonction de transfert optique FTO $(\mu, v)$ comme la transformée de Fourier de la PSF, soit, selon les normes en vigueur :

$$
\operatorname{FTO}(\mu, v)=\int_{-\infty}^{\infty} \int_{-\infty}^{+\infty} \operatorname{PSF}(x, y) e^{-2 \pi i(\mu x+v y)} d x . d y
$$

Expression dans laquelle $\mu$ et $v$ ont la dimension de l'inverse d'une variable d'espace: ce sont des fréquences spatiales qui peuvent s'exprimer en cycles par unité de variable d'espace (longueur ou angle).

Réciproquement, on peut calculer la PSF à partir de la FTO :

$$
\operatorname{PSF}(x, y)=\int_{-\infty}^{\infty} \int_{-\infty}^{\infty} \operatorname{FTO}(\mu, v) e^{+2 \pi i(\mu x+v y) d \mu . d v}
$$

Il est à noter que la FTO est un ensemble de nombres complexes.

\section{1,2.2 Définition de la FTM}

La Fonction de Transfert de Modulation (FTM) est le module de la FTO.

\section{3) Système de coordonnées}

Le fréquence spatiale est une variable à deux dimensions; on la rapporte à deux axes orthogonaux ayant les directions normalisées suivantes :

- tangentielle : un objet périodique a sa fréquence dans la direction tangentielle si elle est constituée de "traits", parallèles à la focale tangentielle d'un système centré entaché d'astigmatisme, c'est-àdire perpendiculaires à la direction menant du point considéré au centre du champ.

- radiale : perpendiculaire à la précédente. 


\section{Propriété fondamentale et conditions d'application}

\section{1) Propriété fondamentale}

Le spectre $\operatorname{SI}(\mu, v)$ de l'image se déduit du spectre de l'objet $\mathrm{SO}(\mu, v)$ et de la FTO par la relation simple :

$$
\operatorname{SI}(\mu, v)=\operatorname{SO}(\mu, v) \cdot \operatorname{FTO}(\mu, v)
$$

Expression valable en nombres complexes (produit des modules et somme des arguments).

\section{2) Conditions d'application}

La propriété fondamentale ci-dessus est applicable aux conditions suivantes :

1) l'objet doit admettre une transformée de Fourier ;

2) le processus d'imagerie doit être linéaire en énergie : en particulier, l'éclairage doit être cohérent ;

3) le processus d'imagerie doit être isoplanétique, c'est-àdire que la FTO ne doit pas dépendre de la position de l'objet dans le champ ;

4) les variables d'espace objet et image doivent être ramenées à la même échelle en tenant compte du grandissement.

\section{Détermination expérimentale}

\section{1) Méthode et principe}

La méthode la plus usuelle comporte trois principales étapes :

1) Présenter au système à tester un objet de spectre connu et aussi étendu que nécessaire :

2) Recueillir l'image et déterminer son spectre, au moins pour les fréquences auxquelles on s'intéresse ;

3) Diviser ce spectre par le spectre de l'objet. Plus le spectre de l'objet sera large et uniforme, plus cette division aura le caractère d'une simple correction.

Cette méthode est le plus souvent mise en œuvre selon l'un des deux principes suivants :

- Balayage séquentiel des fréquences: pour chacune des fréquences désirées. on effectue l'analyse d'une image; l'analyseur 
d'image (ou l'objet), qui assure la sélection de la fréquence, présente alors une certaine complexité, tandis que le calcul est très simple.

- Multiplexage des fréquences : l'objet et l'analyseur d'image sont à large bande, la sélection des fréquences est assurée par le calcul qui devient plus complexe.

\section{Exemples d'instruments}

\section{1) Séquentiel: I'ACOFAM}

L'objet est une fente fine éclairée uniformément en lumière incohérente. Un objectif de microscope reprend l'image fournie par le système à tester et la reforme agrandie sur l'analyseur d'image.

L'analyseur d'image est une mire à découpe sinusoïdale. On démontre que son association avec l'objet est équivalent à celle d'une fente uniforme et d'une mire à transparence sinusoïdale. Un récepteur de flux complète le dispositif.

Le calcul, répété pour chaque zone de la mire correspondant à une fréquence, comprend deux phases:

1) Phase exécutée par l'instrument :

- déterminer le maximum et le minimum du signal

- calculer la modulation dans l'image :

$$
\text { Modulation }=\frac{\max -\min }{\max +\min }
$$

2) Phase exécutée par l'opérateur :

Division par le spectre de l'objet. Si la fente est suffisamment fine, cette phase est inutile.

Remarque : ce procédé ne donne accès qu'à la FTM, c'est-à-dire au module de la FTO, et non à sa phase.

\section{2) Multiplex : hanc EALING}

L'objet est un petit diaphragme circulaire.

L'analyseur d'image est un couteau mobile, complété par un récepteur de flux.

Le calcul, dont les paramètres sont entrés par l'opérateur, comporte les étapes suivantes :

- dérivation du signal : le signal enregistré représente l'ESF, la dérivation le transforme en LSF qui admet une transformée de Fourier 


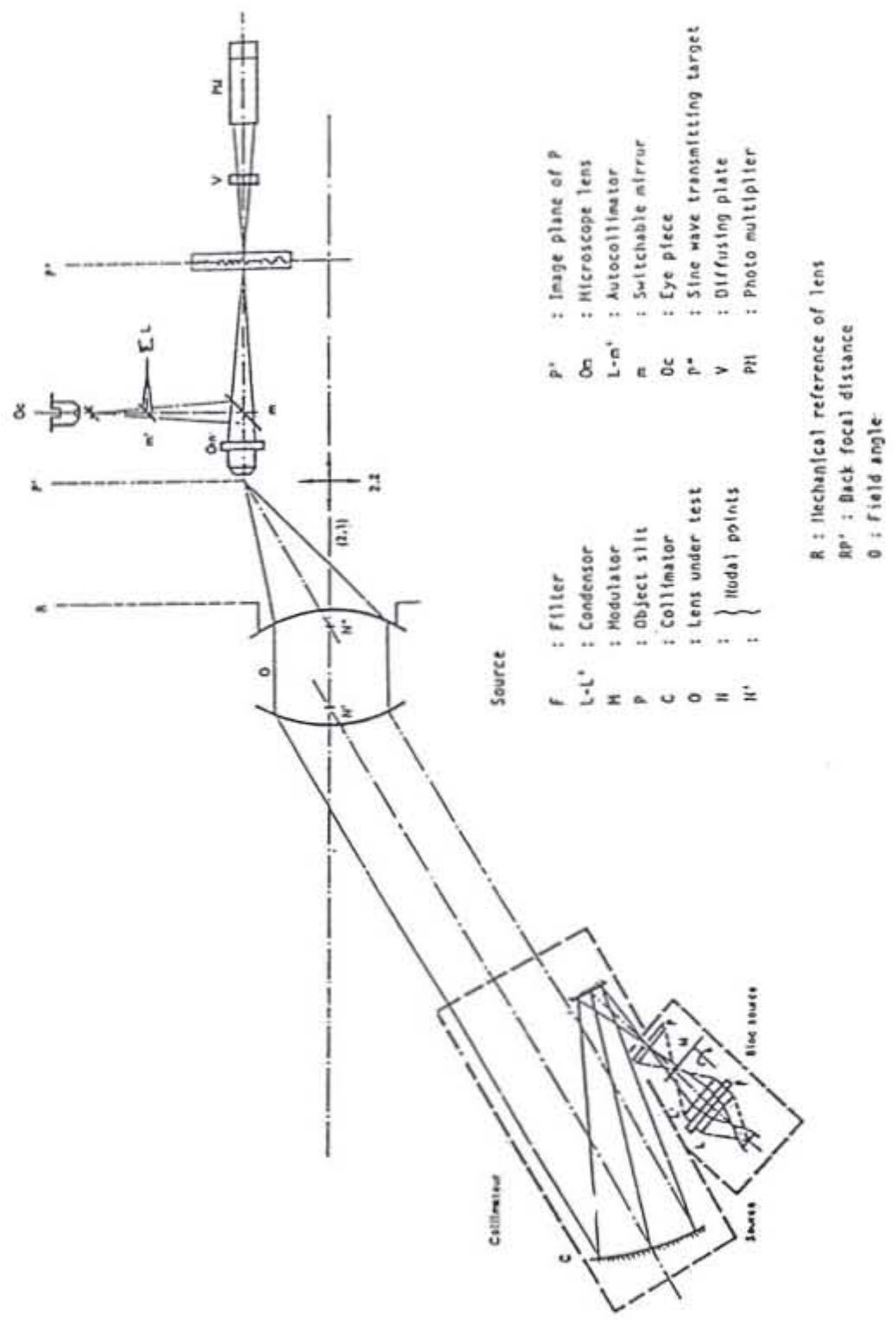

Eiqure 2.1 : ACOFAM bench 


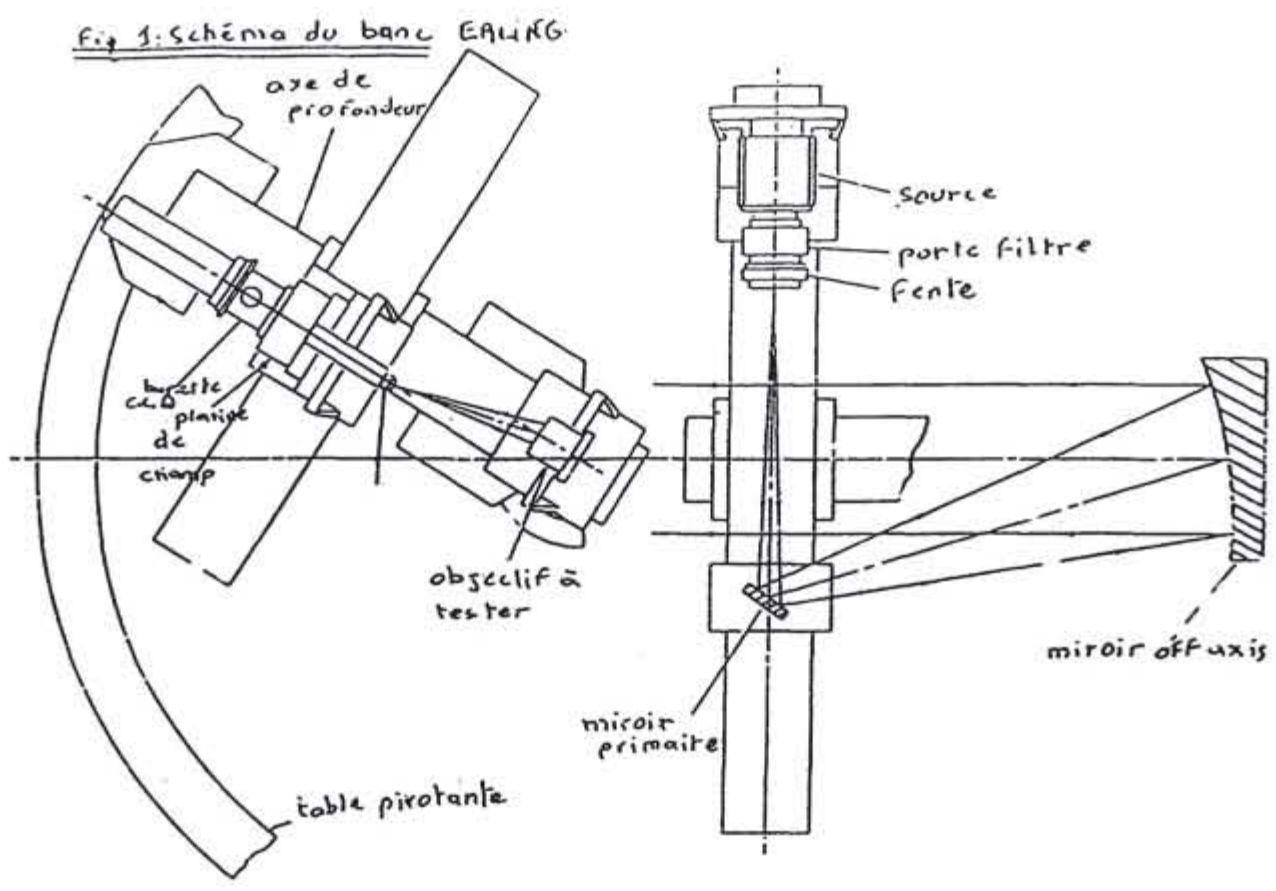

fig. 2.

Tête d'analyse

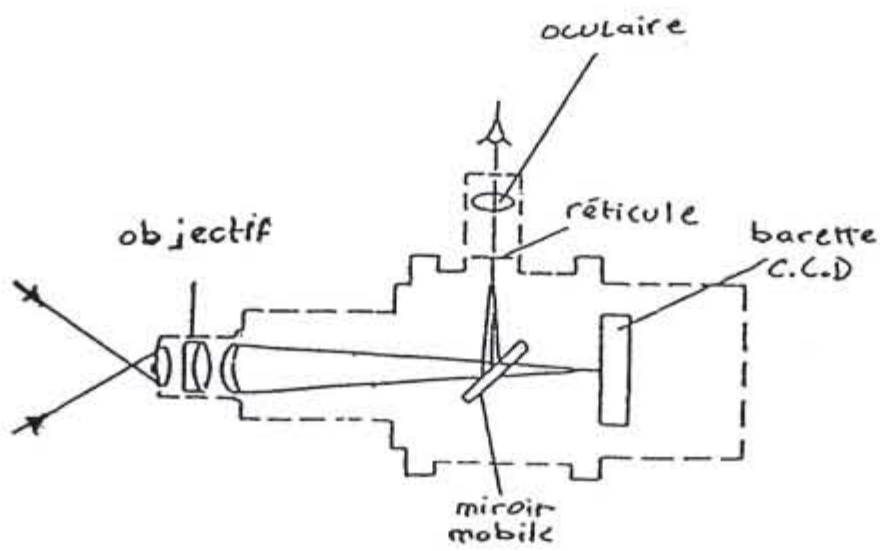


- transformation de Fourier

- mise à l'échelle des fréquences.

Remarques : 1) Ce procédé donne accès à la FTO complète, module et phase.

2) La dérivation est simulée par une différence finie : le résultat est équivalent à celui qu'on obtiendrait en utilisant pour objet une fente de largeur égale au pas d'échantillonnage.

\section{3) Principales précautions}

Un résultat satisfaisant ne peut être obtenu qu'au prix de certaines précautions :

- S'assurer que l'éclairage est bien incohérent, et la chaîne photométrique suffisamment linéaire. Cette précaution est en général du ressort du constructeur de l'instrument.

- Maîtriser suffisamment les paramètres définissant l'état d'imagerie: - composition spectrale du rayonnement

- sensibilité spectrale de la chaîne photométrique

- position de l'objet dans le champ

- choix du plan de la mise au point

- direction d'analyse (radiale, tangentielle, autre)

- S'assurer, dans le cas d'un instrument échantillonnant l'espace-image, que le pas d'échantillonnage est suffisamment petit pour éviter les perturbations dues au repliement du spectre.

- Ramener les fréquences objet et image à la même échelle en tenant compte du grandissement du système sous test.

\section{B) MESURE DE LA DISTORSION D'UN SYSTEME}

\section{OPTIOUE}

Il convient de rappeler tout d'abord quelques notions théoriques, précédemment abordées dans d'autres cours. La distorsion est une aberration de grandissement ; un système de focale $\mathrm{f}$ donne une grandeur 


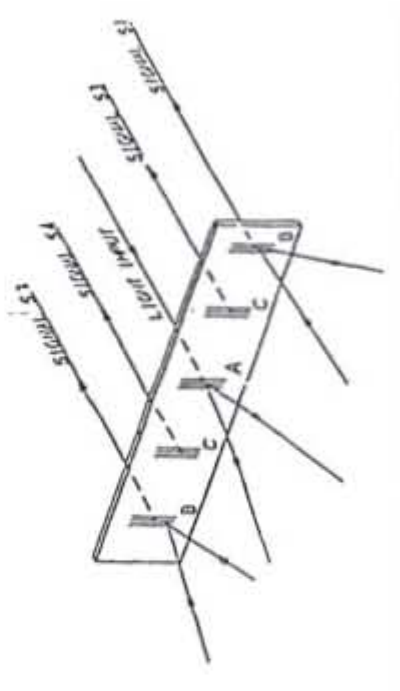

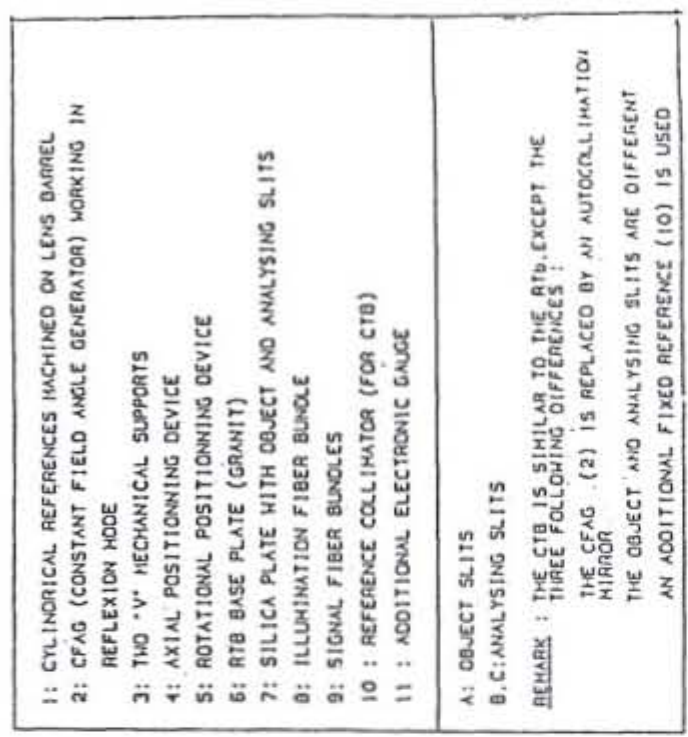
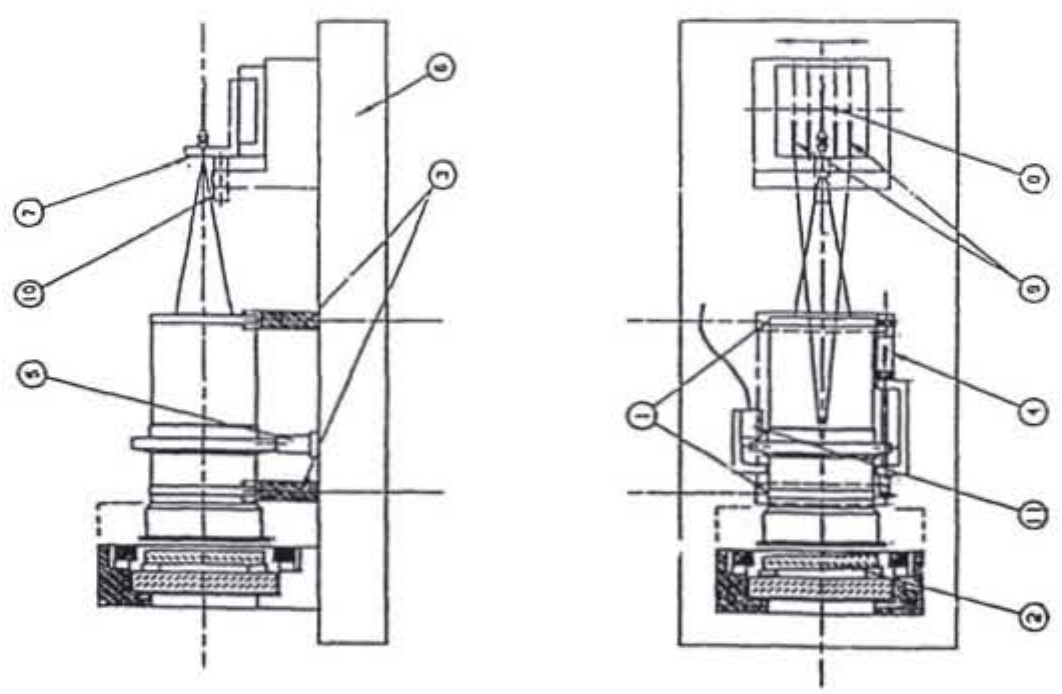

Fiqure 2.2.b : Registration test bench (RTB) 
d'image théorique $I_{t}$, fonction du champ angulaire $\alpha$ selon $I_{t}=f \cdot \tan \alpha$, tandis que l'image réelle à travers l'instrument a une taille $\mathrm{I}_{\mathrm{r}}$.

La distorsion s'exprime alors par le rapport: $\left(\mathrm{I}_{\mathrm{r}}-\mathrm{I}_{\mathrm{t}}\right) / \mathrm{I}_{\mathrm{t}}$

Elle s'exprime habituellement en \%. Quand l'image réelle est plus petite que l'image théorique, on a une distorsion négative dit en " barillet", dans le cas contraire, la distorsion est positive et dite en "coussinet". La distorsion est indépendante de la qualité de l'image dans le principe (cf par exemple les objectifs Fish-Eye : bonne qualité image, mais importante distorsion due au très grand champ). Elle est indépendante de l'ouverture et proportionnelle au cube du champ. Ce caractère original de la distorsion impose des méthodes particulières de mesure.

Pour beaucoup d'objectifs ou d'instruments, la mesure de la distorsion n'est pas demandée, parce que celle-ci est suffisamment faible théoriquement pour ne pas déformer l'image. La mesure s'impose lorsque l'on a besoin de connaitre exactement la grandeur d'image correspondant à un champ angulaire donné, ou le grandissement entre deux plans objetimage. Le premier cas est celui des objectifs de photogrammétrie qui servent à la cartographie. Les objectifs de photographie aérienne ont des grands champs jusqu'à $120^{\circ}$, et des distorsions de l'ordre de $10^{-4}$ (et parfois moins), ce qui représente quelques microns de différence entre l'image théorique et l'image réelle. Le second domaine est la microlithographie permettant l'insolation des circuits imprimés. Les objectifs de grandissement donné (5 ou 10) ont une grande ouverture et un champ relativement important. Ils sont quasi-monochromatiques. En qualité, ils atteignent dans tout le champ des FTM supérieures à 0,5 , pour des fréquences de 500 à $1000 \mathrm{~mm}^{-1}$ selon la longueur d'onde utilisée. La distorsion calculée est inférieure à 0,1 micron dans tout le champ.

Le principe de mesure est tout à fait logique. Il s'agit de mesurer la grandeur d'image correspondant à un angle donné. On peut faire cette mesure avec un collimateur tournant devant l'objectif à tester, la précision relative obtenue sur la distorsion étant la somme des précisions sur l'angle et la taille de l'image. On cherche, dans les procédés modernes, à augmenter cette précision en utilisant des codeurs et des prismes pour obtenir des angles fixes connus très exactement. L'image d'un quadrillage est utilisée assez souvent, auquel cas on mesure la variation de grandissement entre les carrés du bord du quadrillage et ceux du centre. On peut également opérer par photographie. C'est le même 
principe qui sert en microlithographie, mais il est adapté aux valeurs à mesurer. On n'utilise pas un quadrillage, mais des points objets dont les coordonnées sont parfaitement connues. La mesure de la position de l'image de ces points est extrêmement délicate, compte tenu de la précision recherchée (quelques centièmes de microns).

\section{C) MESURE DE LA LUMIERE PARASITE}

Cette mesure est actuellement souvent demandée dans les systèmes optiques destinés à une utilisation spatiale. En effet, le signal principal étant la plupart du temps très faible, une source parasite importante -le soleil, par exemple- peut altérer complètement celui-ci.

La lumière parasite a deux origines principales; premièrement, chaque surface, même soigneusement traitée anti-réflexion, a un coefficient résiduel de réflexion qui varie de 0,1 à $1 \%$, selon les matériaux utilisés et la largeur de la bande spectrale. Après un nombre pair de réflexions (les ordres supérieurs à deux sont généralement sans influence), ce flux parasite se retrouve dans l'image. Le moyen de réduire cette lumière parasite consiste à améliorer l'efficacité des traitements antiréflexion. D'autre part, les rayons hors du champ utile, après réflexion sur les bords des lentilles, des barillets ou de la monture, atteignent finalement l'image. Ces effets de bord créent un flux parasite qui est souvent plus important en valeur absolue que le premier. Les moyens de remédier à cet inconvénient consistent à traiter anti-reflets les montures et les bords , en utilisant des peintures spéciales ou des "pièges " à lumière (filetage ou diaphragme). Quand on dispose de suffisamment de place, on adapte un pare-soleil devant l'objectif, qui limite le faisceau entrant dans l'objectif au faisceau utile nécessaire à la formation de l'image. Pratiquement tous les instruments d'optique spatiale sont équipés ainsi.

Le dispositif de mesure est conforme au schéma ci-après : une sphère intégrante crée un éclairement uniforme dans tout l'espace. Un point noir placé au foyer d'un miroir concave donne une image à l'infini 

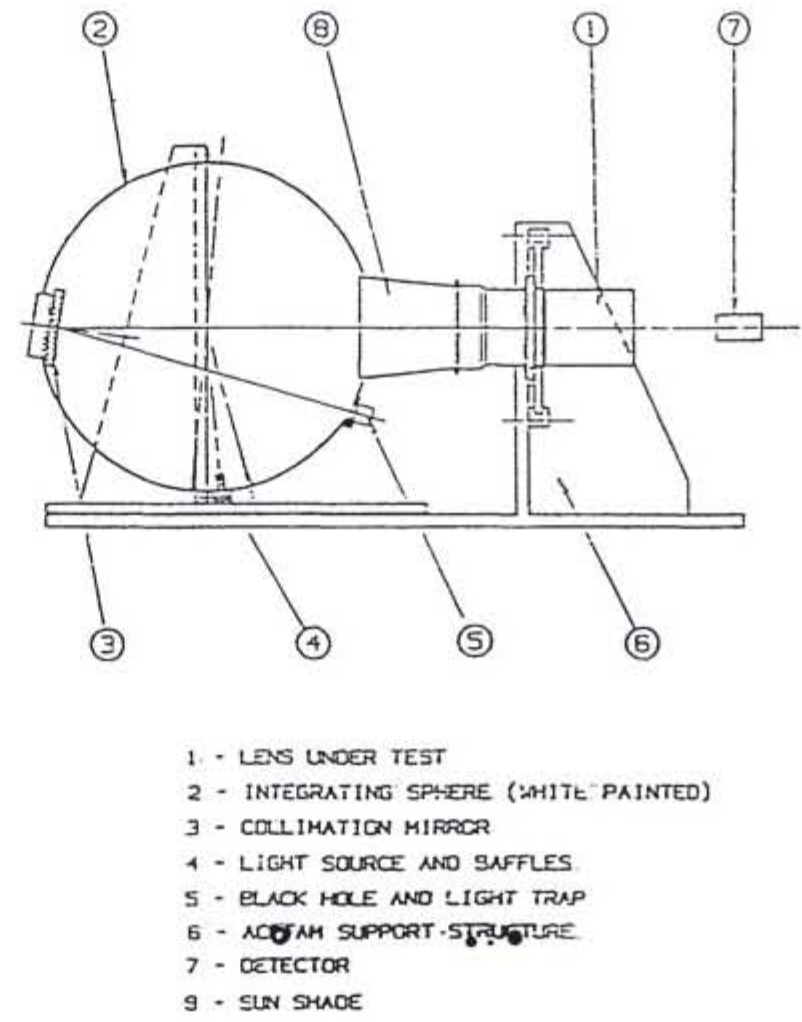

Figure 2.4 : Veiling glare bench (VGB) 
qui se retrouve donc au foyer de l'objectif à tester, dont la pupille d'entrée est à l'intérieur de la sphère. Dans le plan focal de l'objectif, on mesure l'éclairement $E_{0}$ dans l'image du point noir, et de part et d'autre de celui-ci ( $E_{1}$ et $E_{2}$ ). Après étalonnage du zéro, on obtient le pourcentage de lumière parasite par le rapport :

$$
t=E_{0} /\left(\left(E_{1}+E_{2}\right) / 2\right)
$$

\section{ANNEXE 1: SURFACES ASPHERIOUES}

Les surfaces asphériques ont été utilisées depuis longtemps dans les miroirs de téléscopes destinés à l'ohservation astronomique. Ces miroirs étaient souvent des coniques, paraboloïde et hyperboloïde dans le cas du Ritchey Chrétien. Ils étaient réalisés par retouches locales à partir d'une surface sphérique, compte tenu de leur grand diamètre. Ces miroirs sont fabriqués à l'époque actuelle par des procédés assez analogues, par des sociétés spécialisées. De nouvelles méthodes, par dépôt à partir d'une surface sphérique de manière à réaliser un profil donné, ont été mises au point. Elles sont limitées par l'épaisseur du dépôt.

L'usage des surfaces asphériques s'est développé considérablement avec les matériaux infra-rouge. En effet, la qualité de l'image à des grandes longueurs d'onde permet d'accepter une qualité de surface compatible avec l'utilisation des machines automatiques à usinage diamant. Dans ce cas, la surface asphérique de révolution est créé par commande numérique. Dans le domaine visible, le verre est usiné par micro-rectification, et il est souvent nécessaire de faire un polissage après usinage du profil. Tout l'art consiste à ne pas détériorer celui-ci dans cette opération. Ces dernières années ont été élaborées des méthodes par moulage, qui peuvent avoir un avenir intéressant.

Le contrôle de ces surfaces est fait par le fabricant lors de l'usinage. Il est réalisé par palpage, ce qui permet une mesure directe à 
0,2 micron près, ou parfois par contrôle au ZYGO, après mise place d'un correcteur.

\section{ANNEXE 2: L'INTEREEROMETRE ZYGO}

Celui-ci se compose de deux parties : le boîtier principal, et le ZAPP " ZYGO AUTOMATIC PATTERN PROCESSOR ".

\section{A) LE BOITIER PRINCIPAL}

Le boîtier est le cœeur du système de mesure. Il comprend non seulement les éléments communs à la plupart des interféromètres tel que le laser et son alimentation, et un objectif de collimation, mais également une caméra vidicon, un zoOM de rapport 6 , un système d'auto-alignement et un dispositif d'acquisition rapide des franges. Un moniteur vidéo permet de voir les réseaux de franges ou d'en faire l'acquisition.

\section{B) LE “ ZAPP"}

Il se compose de différents éléments : un console, un moniteur, un calculateur, une imprimante, un écran pour prise de vue, et une caméra pour la lecture d'interférogrammes.

Lorsque les réglages sont terminés, le réseau de franges apparaît sur le moniteur. Le ZAPP est alors prêt à acquérir automatiquement les coordonnées des centres des franges dans la zone à mesurer, qui peut s'ajuster à volonté par quatre curseurs délimitant une fenêtre. Le ZAPP mesure alors les centres des franges. Ceux-ci sont visualisés sur l'écran de contrôle par des courbes de $\times$ et + (voir photo). L'opérateur peut alors contrôler si les données acquises sont suffisantes, ou s'il est nécessaire d'en supprimer ou au contraire d'en rajouter de nouvelles, par agrandissement de la fenêtre de mesure par exemple.

A ce stade, le calculateur détermine par une méthode des moindres carrés les défauts Pic to Valley et RMS de la surface à mesurer. 


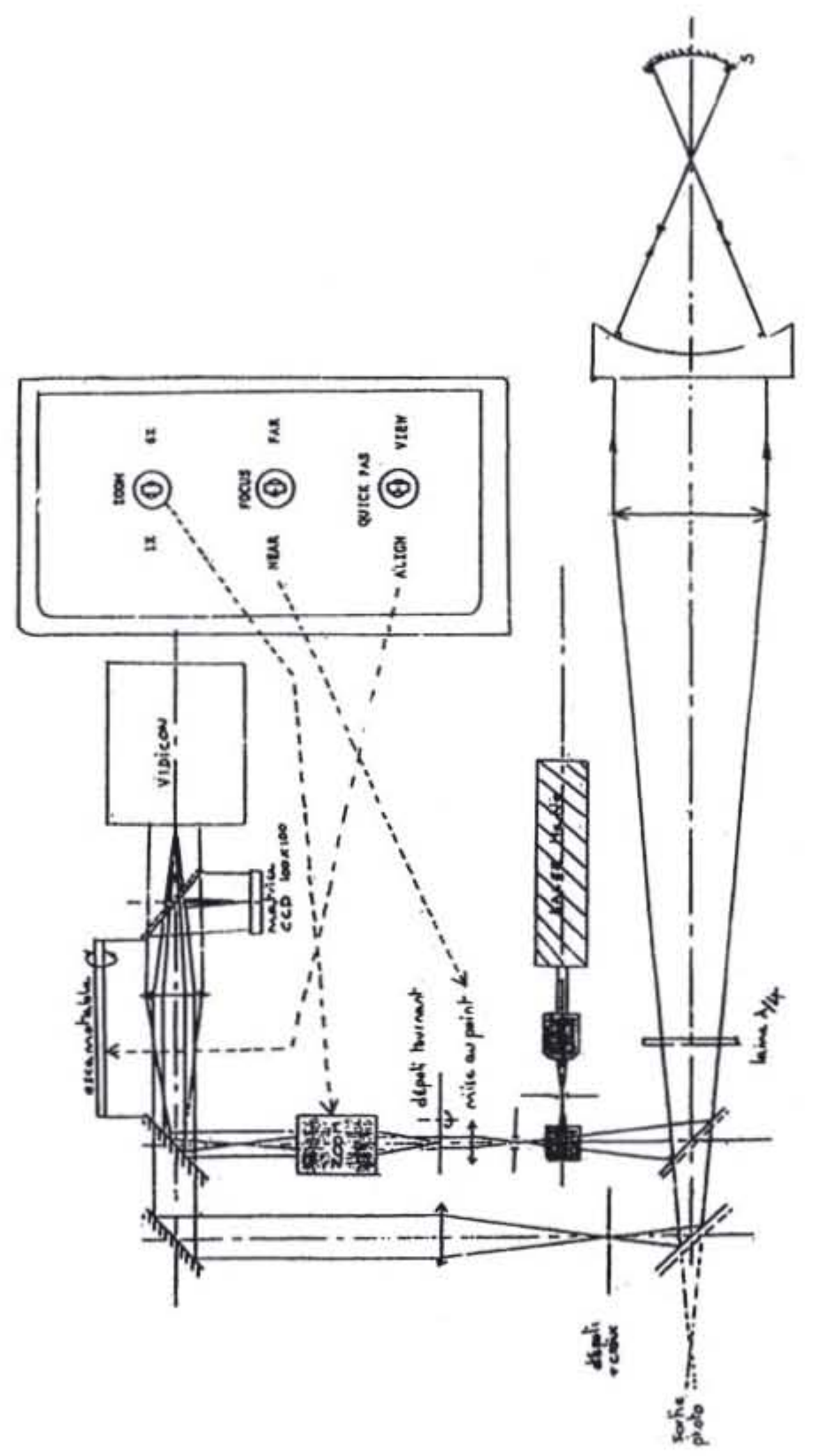


$220 \quad P$. Coussot
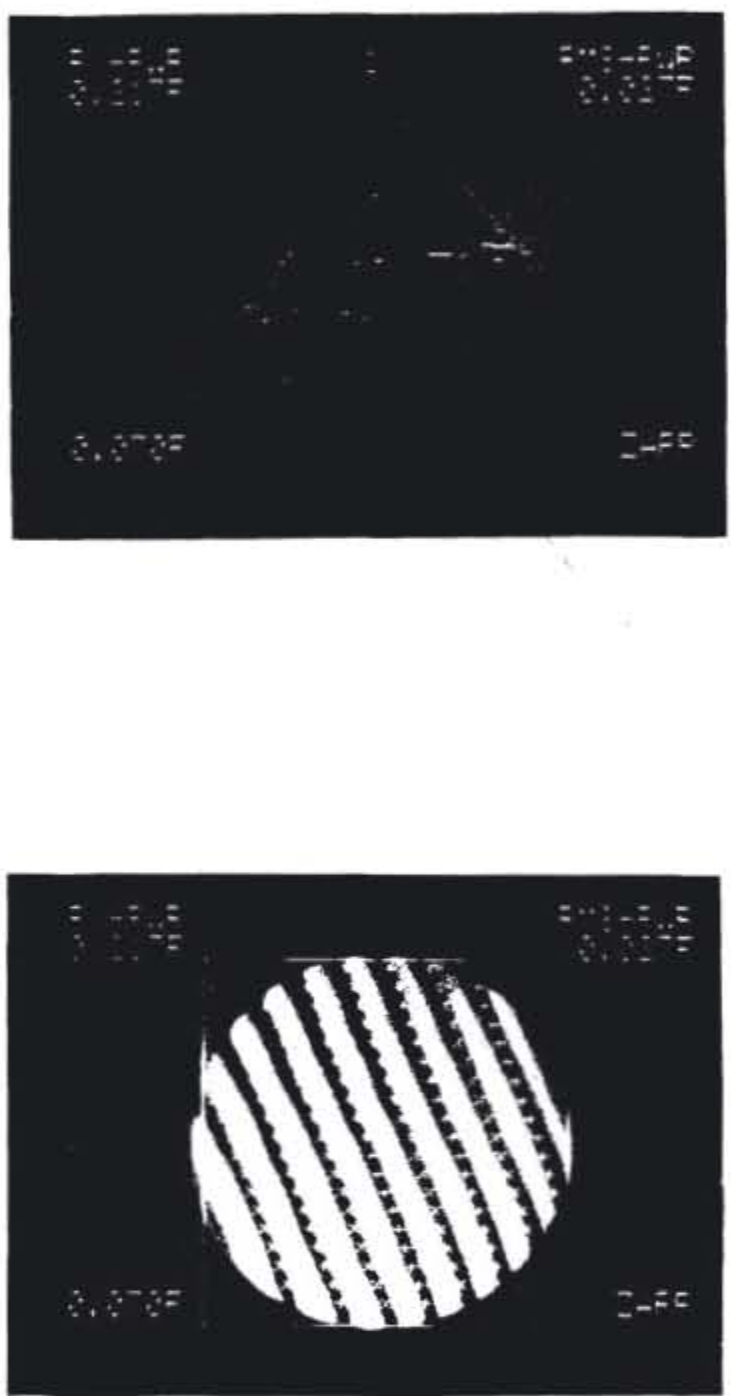


\section{C) L,FS POSSIBILITES DU ZYGO}

Elles sont nombreuses :

- vérification de la planéité des surfaces planes,

- mesure de la surface d'onde en transmission et du parallélisme d'une lame,

- mesure du rayon de courbure et des défauts d'irrégularité de surface concaves et convexes (en utilisant des objectifs additionnels),

- évaluation de la performance d'un système complet en surface d'onde.

Enfin, il est possible d'obtenir l'interférogramme d'un système optique complet. Par couplage avec le programme CODE $\mathrm{v}$, on analyse les aberrations résiduelles de révolution et d'excentrements, et par le calcul le programme détermine les variations de déplacement (suivant les trois axes) des éléments susceptibles de corriger en totalité ou partiellement les défauts. 